\title{
Simulation des grandes échelles d'un aspirateur de centrale hydraulique
}

\author{
Cédric Duprat ${ }^{1}$, Gulllaume Balarac ${ }^{1}$, ${\text { Olivier } \text { Métais }^{1} \text { et Thomas Laverne }}^{2}$ \\ 1 Laboratoire des Écoulements Géophysiques et Industriels (LEGI), 1024 rue de la Piscine, 38041 Grenoble, France \\ 2 Alstom Hydro France, Établissement de Grenoble, 82 Avenue Léon Blum, BP75, 38041 Grenoble \\ Cedex 9, France
}

Reçu le 20 avril 2009

Résumé - La technique de simulation des grandes échelles (SGE) est utilisée pour simuler l'écoulement dans un aspirateur de centrale hydraulique à différents points de fonctionnement. La complexité de la géométrie et le haut nombre de Reynolds de l'écoulement nécessitent l'utilisation de méthodes spécifiques. Ainsi, nous avons développé un traitement à la paroi tenant compte à la fois du frottement pariétal mais aussi du gradient de pression. La technique utilisée pour permettre de générer des conditions d'entrée instationnaires réalistes, à partir de données expérimentales, est également détaillée. Les différents régimes d'écoulements résultant de cette configuration sont alors présentés.

Mots clés : SGE / loi de paroi / condition d'entrée turbulente / hydroélectricité

\begin{abstract}
Large eddy simulation of a complex hydroturbine draft tube. Large eddy simulation method (LES) has been used to simulate flow motion throught a draft-tube of hydraulic power plant at different operating points. Given the high Reynolds number and the complex geometry, specific methods need to be used. Thus, a new wall model has been developped to take into account both shear stress and pressure gradient effects. The turbulent inflow boundary condition based on experimental data has been also detailled. Different operating points of this configuration have been computed and results are presented.
\end{abstract}

Key words: LES / wall model / turbulent inflow / hydroelectricity

\section{Introduction}

La complexité de la géométrie d'un aspirateur de centrale hydraulique, organe reliant la turbine au bassin aval de la centrale (voir Fig. 1), entraîne un écoulement turbulent complexe. L'écoulement est caractérisé par un nombre de Reynolds basé sur le rayon de la turbine et la vitesse débitante de l'ordre de $10^{6}$. Les gradients adverses de pression en aval de la turbine peuvent entraîner des décollements et des phénomènes tourbillonnaires plus complexes. Malgré de nombreuses études expérimentales $[1,2]$ et numériques $[3,4]$, l'analyse de l'écoulement dans un aspirateur reste un enjeu. L'objectif de cette étude est d'analyser l'écoulement en utilisant un modèle de simulation des grandes échelles (SGE). Celui-ci est connu pour être mieux adapté à l'étude d'écoulements fortement instationnaires que les modèles moyennés usuellement utilisés dans l'industrie.

Cependant l'utilisation de la SGE pour traiter des écoulements industriels nécessite le développement de méthodes spécifiques qui ne sont pas encore des standards de codes CFD commerciaux classiques.

Tout d'abord, la présence de paroi contraint fortement la discrétisation, rendant ainsi inaccessible à la SGE des configurations industrielles. Pour s'affranchir de cette contrainte, la région proche paroi peut être traitée soit en résolvant une équation simplifiée de l'écoulement à la paroi (TBLE, méthode hybride RANS/LES), soit en imposant une condition algébrique reliant directement la vitesse à la distance à la paroi (loi de paroi). Nous proposons dans ce travail, une loi de paroi prenant en compte le gradient de pression.

Comme la SGE calcule explicitement une part des instationnarités de l'écoulement, les conditions d'entrée doivent les décrire. Nous présenterons ainsi une façon de générer des conditions d'entrée turbulentes pour illustrer l'écoulement à la sortie de la turbine à partir de mesures expérimentales [5]. 


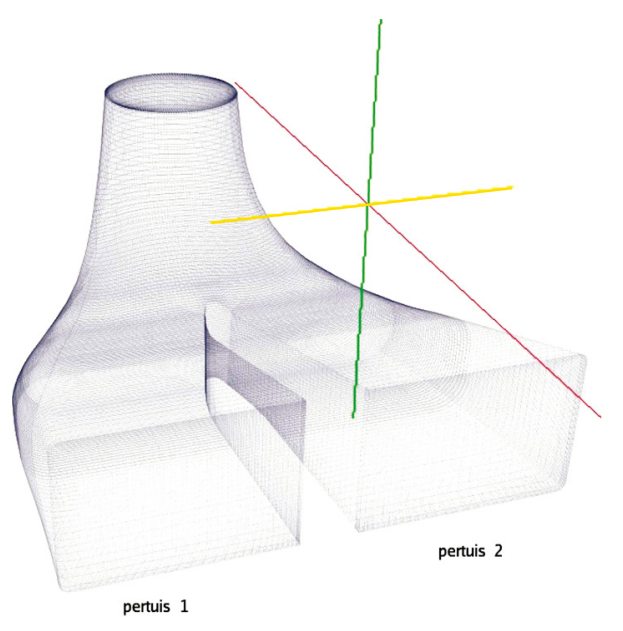

Fig. 1. Géométrie réelle de l'aspirateur.

\section{Méthodes}

\subsection{Méthodes numériques}

Le code utilisé est OpenFOAM, un logiciel libre de CFD basé sur des classes C++ (http://www.opencfd. co.uk). Il permet de résoudre les équations de NavierStokes incompressibles par la méthode des volumes finis. On utilise pour discrétiser ces équations des schémas centrés d'ordre deux à la fois en espace et en temps. La décomposition du domaine permet d'effectuer des calculs en parallèle.

Le principe de la méthode SGE est de résoudre explicitement les grandes échelles du mouvement et modéliser les plus petites par un modèle sous maille. L'implémentation des techniques SGE dans OpenFOAM a été testée et validée [6]. Parce que le maillage sera faiblement résolu dans la direction de l'écoulement, il apparait plus adapté d'utiliser un modèle sous-maille avec une équation de transport pour l'énergie cinétique sous-maille [7]. La condition de sortie, adaptée à la SGE, permet de s'assurer que les tourbillons traversant la sortie ne perturbent pas le domaine de calcul. Nous avons utilisé une condition convective du type Orlanski [8] qui satisfait ces conditions. Une extension de la géométrie (Fig. 2) permet d'uniformiser l'écoulement en sortie de l'aspirateur. La géométrie contient $1,9 \times 10^{6}$ nœuds, dont la répartition est raffinée à la paroi. Malgré cette précaution, le gradient moyen ne peut être résolu à la paroi pour un nombre de Reynolds si élevé. L'utilisation de méthode adaptée comme les lois de paroi est alors nécessaire. La loi de paroi permet ainsi de modéliser la couche limite et le gradient moyen non résolu.

\subsection{Conditions à la paroi}

Afin de s'affranchir de contraintes de discrétisation trop importantes, nous modélisons l'écoulement proche de la paroi par une expression analytique. Celle-ci relie, à proximité de la paroi, la composante tangentielle

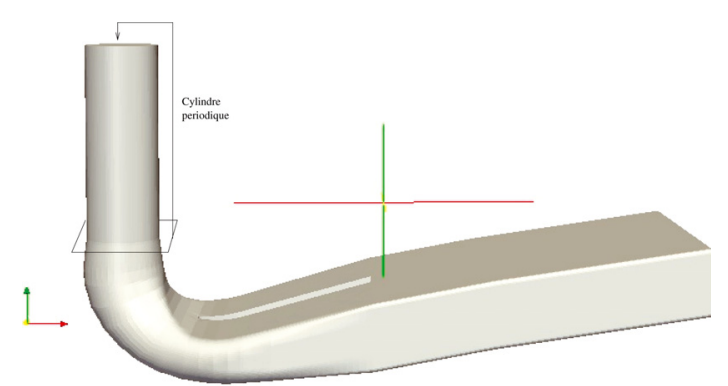

Fig. 2. Géométrie de calcul.

de la vitesse à la distance à la paroi. La loi de paroi usuellement utilisée fait l'hypothèse d'une couche limite en équilibre. Cette hypothèse n'est plus valable avec des géométries complexes, où les gradients de pression viennent déstabiliser la couche limite. Nous avons ainsi développé une nouvelle loi de paroi prenant en compte le gradient de pression. La formulation présentée est validée a priori pour des écoulements attachés et décollés [9].

Le bilan de quantité de mouvement longitudinale pour l'écoulement moyen peut se simplifier à proximité de la paroi [10] par,

$$
\frac{\partial U}{\partial t}+\frac{\partial U V}{\partial y}=-\frac{1}{\rho} \frac{\partial p}{\partial x}+\nu \frac{\partial^{2} U}{\partial y^{2}}-\frac{\partial\left\langle u^{\prime} v^{\prime}\right\rangle}{\partial y}
$$

où $\nu$ et $\rho$ sont respectivement la viscosité et la masse volumique du fluide, $U$ et $V$ sont les vitesses moyennes longitudinale et normales à la paroi et $y$ est la direction normale à la paroi. Le dernier terme représente les contraintes de Reynolds. Pour fermer cette équation on utilise l'hypothèse de viscosité turbulente qui modélise l'effet dissipatif des termes du tenseur de Reynolds. Parmi les termes de non-équilibre, susceptible de déstabiliser la couche limite, seul le gradient de pression est supposé non-négligeable. Ces hypothèses permettent d'écrire l'équation (2).

$$
0=\nu \frac{\partial^{2} U}{\partial y^{2}}+\frac{\partial}{\partial y}\left(\nu_{t} \frac{\partial U}{\partial y}\right)-\frac{1}{\rho} \frac{\partial p}{\partial x}
$$

L'équation (2) peut s'intégrer une première fois, en utilisant la définition de la contrainte pariétale $\tau_{w}=$ $\left.\nu \rho \frac{\partial U}{\partial y}\right|_{y=0}$ :

$$
0=\left(\nu+\nu_{t}\right) \frac{\partial U}{\partial y}-\frac{1}{\rho} \frac{\partial P}{\partial x} y-\frac{\tau_{w}}{\rho}
$$

La notation classique adimensionnelle utilise une vitesse de frottement $u_{\tau}=\sqrt{\frac{\tau_{w}}{\rho}}$, pour définir la longueur et la vitesse adimensionnelle $y^{+}=\frac{y u_{\tau}}{\nu}$ et $U^{+}=\frac{U}{u_{\tau}}$. Seulement, à proximité d'un point de décollement, le frottement et donc $u_{\tau}$ sont nuls. Ces points, courant dans les écoulements complexes, ne permettent pas d'utiliser la notation classique. On utilisera donc la notation définie par Manhart [11] qui propose un nouveau dimensionnement :

$$
y^{*}=\frac{y u_{\tau p}}{\nu} \quad U^{*}=\frac{U}{u_{\tau p}}
$$


où $u_{\tau p}=\sqrt{u_{\tau}^{2}+u_{p}^{2}}$ est une nouvelle vitesse caractéristique avec, $u_{p}=\left|\frac{\mu}{\rho} \frac{\partial P}{\partial x}\right|^{1 / 3}$. Un paramètre $\alpha=\frac{u_{\tau p}^{2}}{u_{p}^{2}}$ permet de quantifier laquelle des contributions entre celle $\mathrm{du}$ gradient de pression et celle du frottement est la plus importante. L'équation (3) peut donc se réécrire sous la forme adimensionnelle suivante :

$$
\frac{\partial U^{*}}{\partial y^{*}}=f\left(\alpha, y^{*}, \frac{\nu_{t}}{\nu}, \operatorname{sign}\left(\tau_{w}\right), \operatorname{sign}\left(\frac{\partial P}{\partial x}\right)\right)
$$

La comparaison des signes du gradient de pression et de la contrainte de frottement permet de caractériser le gradient de pression favorable ou adverse au mouvement global des forces d'inertie. On utilise les travaux de Nituch et al. [12] et de Balaras et al. [10] pour définir la viscosité turbulente de la façon suivante :

$$
\frac{\nu_{t}}{\nu}=\kappa y^{*}\left(\alpha+y^{*}(1-\alpha)^{3 / 2}\right)^{0,78}\left(1-\mathrm{e}^{-\frac{y^{*}}{1+A \alpha^{3}}}\right)^{2}
$$

où $A=17$ et $\kappa$ est la constante de von Kàrmàn.

Ainsi, les équations (5) et (6) constituent le modèle à la paroi, validé a priori [9], utilisé pour les simulations des aspirateurs présentées par la suite.

La vitesse caractéristique locale de l'écoulement $u_{\tau p}$ est déduite des deux dernières équations par un algorithme de Newton-Raphson. La vitesse caractéristique du gradient de pression, $u_{p}$, est connue par le calcul ce qui permet de déduire la vitesse de frottement $u_{\tau}$. Le gradient de vitesse issu du calcul est corrigé par une viscosité, dans la première maille, calculée par la contrainte de frottement $\tau_{w}$ issue de la loi de paroi.

\subsection{Condition d'entrée}

Dans le cas de simulations basées sur des méthodes statistiques, un profil moyen analytique ou expérimental peut être imposé pour les variables caractérisant la turbulence. Pour la SGE, l'écoulement en entrée doit être turbulent c'est-à-dire décrit par un signal instationnaire. Une méthode est de faire converger un calcul annexe et de l'utiliser pour fournir à chaque pas de temps un champ d'entrée pleinement turbulent. On utilisera dans notre cas un conduit périodique. L'écoulement en sortie de la géométrie périodique est donc à la fois utilisé comme condition d'entrée pour l'aspirateur et réinjecté en entrée (Fig. 2). Des termes de forcage sont ajoutés aux équations de Navier-Stokes pour conserver un débit et un taux de rotation constant [13]. Ainsi, une force axiale permet de compenser le frottement à la paroi et d'atteindre le débit fixé par l'utilisateur. Le traitement de la force orthoradiale est proposé par Landman [14] sous la forme suivante :

$$
f_{\theta}(r)=F\left[1+\left(\frac{r}{R}\right)^{2}\right]^{-2}
$$

où $R$ est le rayon du conduit et $F$ l'amplitude de la force à imposer pour satisfaire le nombre de swirl recherché.

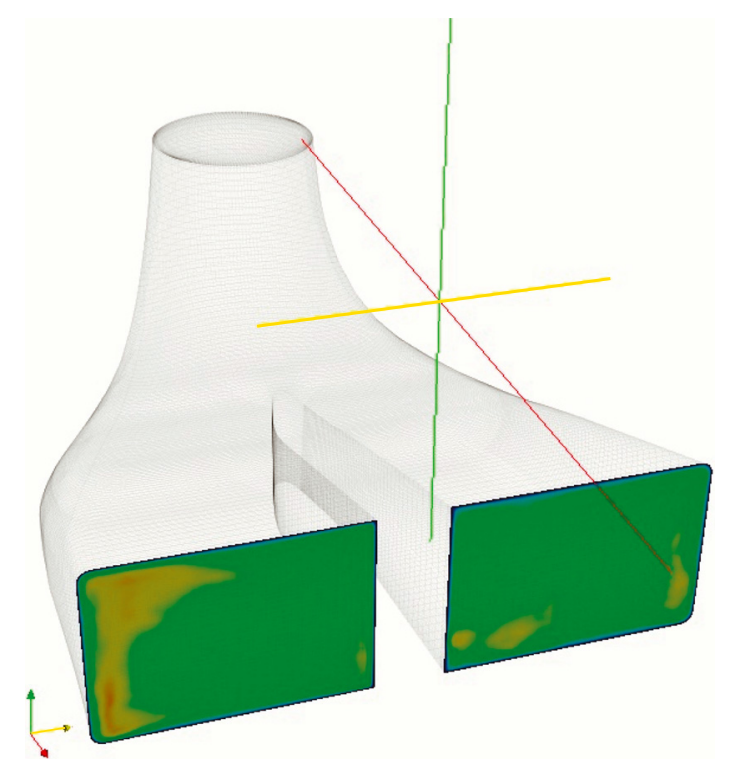

Fig. 3. Coupe de vitesse moyenne en sortie des pertuis de l'aspirateur.

On définit le nombre de swirl dans le repère orthoradial $\left(\boldsymbol{e}_{\boldsymbol{r}}, \boldsymbol{e}_{\boldsymbol{\theta}}, \boldsymbol{e}_{\boldsymbol{z}}\right)$ comme le ratio entre le flux de quantité de mouvement angulaire et le flux de quantité de mouvement axial, pondéré par le débit :

$$
S_{w}=\frac{1}{R} \frac{\int_{0}^{R} r^{2}\left|u_{\theta}\right| u_{z} \mathrm{~d} r}{\int_{0}^{R} r u_{z}^{2} \mathrm{~d} r}
$$

Ces deux paramètres sont issus de données expérimentales et représentent une façon simple de modéliser l'écoulement en sortie d'une turbine.

\section{Validations et résultats}

Les résultats sont présentés en deux parties. D'abord, une discussion qualitative de l'écoulement dans le diffuseur au travers de coupes 2D. On détaillera en particulier les mesures de débit dans chaque pertuis. Ces résultats seront confrontés aux mesures expérimentales réalisées en PIV. Ensuite, nous utiliserons des visualisations de structures cohérentes 3D issues de deux critères différents. On s'appuiera sur ces visualisations pour expliquer les phénomènes de l'écoulement à l'aval du coude de l'aspirateur.

L'écoulement dans les pertuis peut se décomposer comme la combinaison de l'écoulement tournant en sortie de la roue et les deux tourbillons contra-rotatifs reflétant l'influence de la courbure du coude. On observe ainsi l'influence des gradients de pression, tant adverses au niveau de la paroi concave, que favorables au niveau de la paroi convexe. Ceci confirme donc que le gradient de pression est un critère à prendre en compte dans la simulation, responsable des décollements et donc des instabilités de la couche limite. Les figures 3 et 4 représentent, à la même échelle, les vitesses instantanées (Fig. 3) et moyennes 


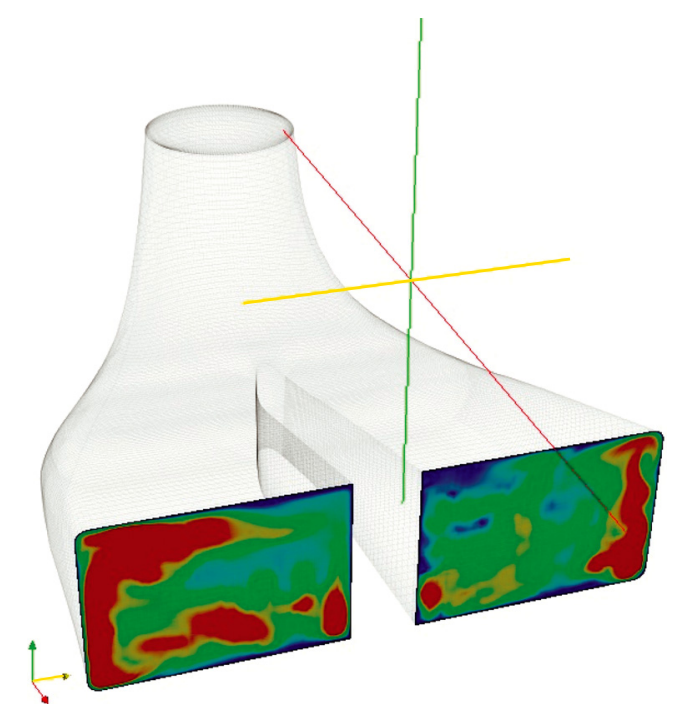

Fig. 4. Coupe de vitesse instantanée en sortie des pertuis de l'aspirateur.

(Fig. 4) en sortie des pertuis pour un premier régime, noté PF1. Le champ instationnaire montre la complexité de l'écoulement et la présence de forts gradients de vitesse dans la section de sortie de l'aspirateur. Le champ moyenné permet d'observer un déséquilibre de débit dans les deux canaux ce qui confirme des résultats déjà établis, à la fois numériquement [3] et expérimentalement [5]. Ces observations du déséquilibre des débits ne se retrouvent pas pour tous les régimes d'écoulements. Ainsi dans un second cas, noté PF2, correspondant à un débit plus faible et un nombre de swirl plus élevé, une répartition uniforme des débits est observée (Cf. tableau ci-dessous)

\begin{tabular}{ccc}
\hline & Pertuis 1 (en \%) & Pertuis 2 (en \%) \\
\hline PF1 & 59 & 41 \\
PF2 & 52 & 48
\end{tabular}

Cette observation confirme des mesures expérimentales PIV dans les pertuis où la répartition de débit est de 60$40 \%$ [5], pour la configuration d'écoulement correspondant au point de fonctionnement PF1. Le calcul étant instationnaire, on peut représenter une évolution temporelle du débit dans chaque pertuis pour les différents régimes de fonctionnement. En abscisse de la figure 5, le temps représente $0,06 \mathrm{~s}$ de l'écoulement physique ce qui est faible pour en déduire un comportement général. Néanmoins, les observations expérimentales et les premières tendances laissent suggérer des oscillations basses fréquences.

La figure 6 représente une visualisation des structures cohérentes de petites tailles présentes dans l'écoulement. Ces structures sont visibles par des iso-valeurs positives du critère $Q$, second invariant du tenseur des gradients de vitesse. Malgré un grand nombre de structures, induit par la valeur importante du nombre de Reynolds, le tourbillon de sortie de la roue est visible en entrée de l'aspirateur. Ce tourbillon est de faible amplitude car au régime considéré le nombre de swirl est faible. La répartition des petites structures est équilibrée dans les deux pertuis. Pour filtrer les plus petites structures, et ne montrer que les structures

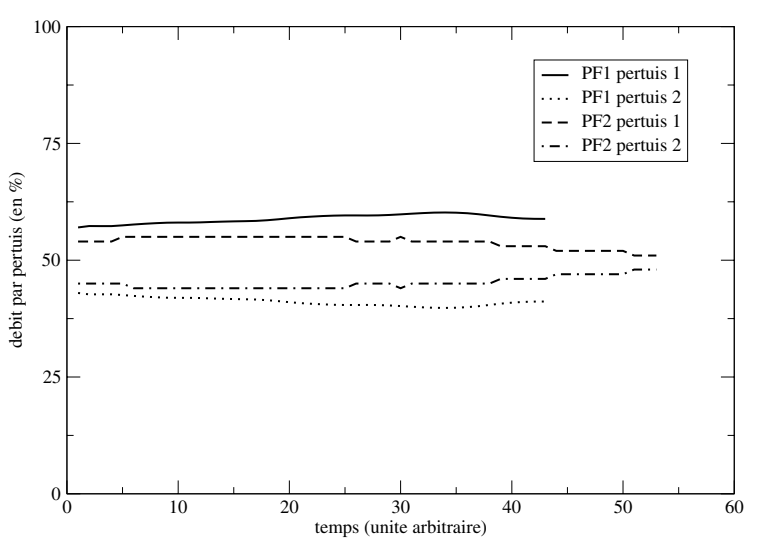

Fig. 5. Évolution des débits instantanés dans les deux pertuis pour le point de meilleur rendement.

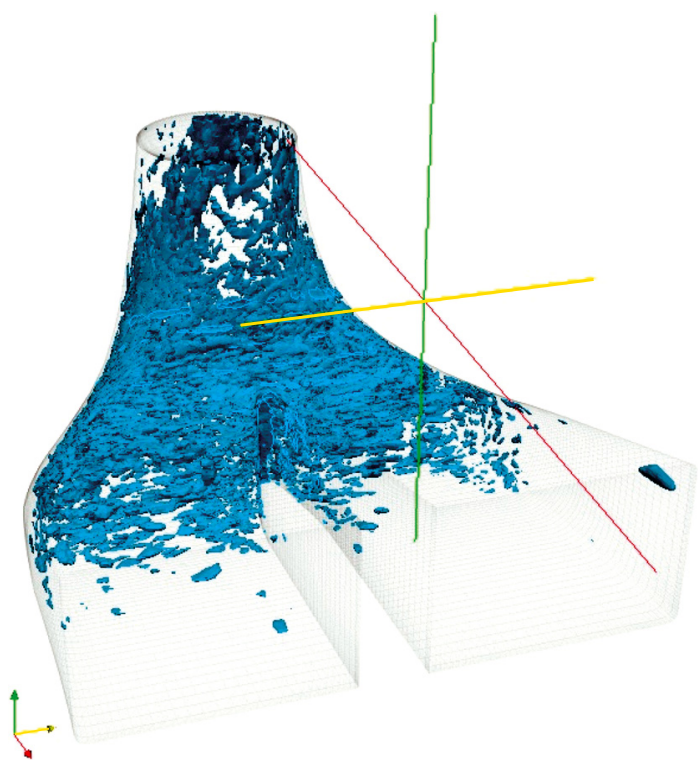

Fig. 6. Visualisation des structures cohérentes par le contour d'iso-Q.

de plus grandes échelles, des iso-valeurs de basse pression sont représentées dans la figure 7. Ainsi, le tourbillon de sortie de roue est de nouveau visible. D'autre part, la répartition dans les pertuis est maintenant déséquilibrée. L'absence de grandes structures dans le pertuis 2 traduit une pression plus importante dans celui-ci. Ces observations des structures cohérentes corroborent avec les mesures faites sur les débits. La présence d'une structure tourbillonnaire dans le pertuis 1 implique une survitesse comme l'illustre la figure 8 , qui montre les vecteurs vitesses moyens. Des études seront poursuivies pour comprendre le développement de cette asymétrie de l'écoulement.

\section{Conclusions}

Les simulations numériques que nous avons menées, ont montré l'écoulement instationnaire dans une 


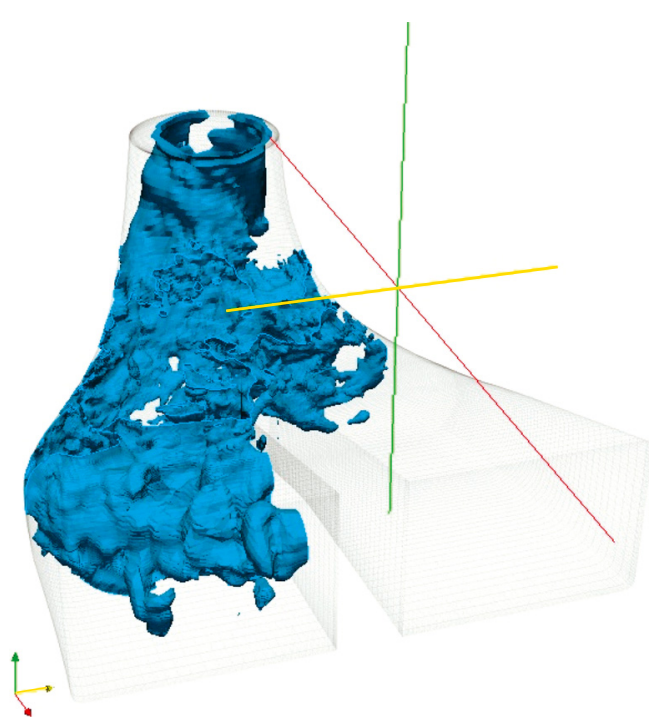

Fig. 7. Visualisation des structures cohérentes par le contour d'iso-pression.

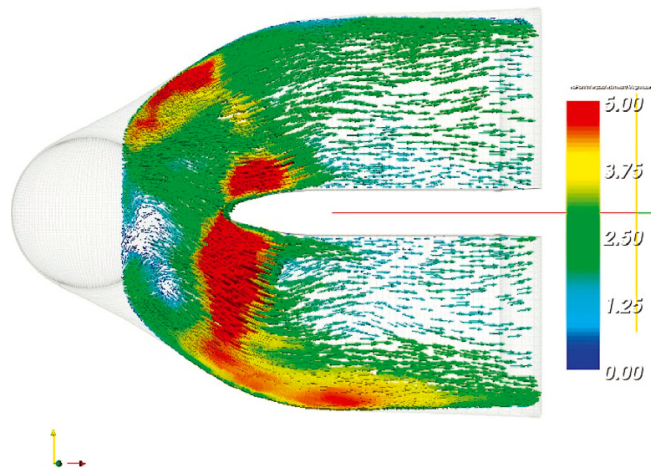

Fig. 8. Vecteurs des vitesses moyennées dans les pertuis colorés par la norme de la vitesse.

géométrie complexe à des nombres de Reynolds utilisés dans l'industrie. Nous avons retrouvé des phénomènes observés expérimentalement mais peu documentés, comme l'asymétrie de l'écoulement en sortie de l'aspirateur. L'utilisation de la SGE pour modéliser la turbulence nous a permis de faire des mesures instationnaires du débit, mettant ainsi en évidence le caractère fluctuant de la répartition de débit au travers des pertuis. Ces phénomènes complexes sont retrouvés en fixant uniquement une valeur de débit et un nombre de swirl en entrée ce qui pourrait correspondre aux deux paramètres importants lors de la conception d'une nouvelle turbine. Pour tester cette hypothèse, d'autres séries de simulations utilisant des conditions d'entrée plus complexes et plus réalistes sont en cours. Elles permettront d'analyser l'influence des conditions d'entrée sur l'écoulement dans l'aspirateur et sur les instabilités présentes.
Remerciements. Les auteurs remercient l'ADEME (Agence de l'environnement et de la maîtrise de l'énergie) et Alstom Hydro France pour leurs soutiens.

\section{Références}

[1] J. Arpe, F. Avellan, Pressure wall mesurements in the whole draft tube: Steady and unsteady analysis, proceedings of 21st IAHR Symp, on Hydraulic Machinery and Systems, Delft, The Netherlands, 2002

[2] M. Iliescu, G.D. Ciocan, F. Avellan, 2 phase PIV measurements at the runner outlet in a francis turbine, FED SM2003-45756, ASME, New York, 2003

[3] J. Paik, F. Sotiropoulos, M.J. Sale, Numerical simulation of swirling flow in a complex hydroturbine draft tube using unsteady statistical turbulence models, J. Hydraulic Engineering 131 (2005) 441-455

[4] R.K. Zhang, F. Mao, J.Z. Wu, S.Y. Chen, Y.L. Wu, S.H. Lui, Characteristics and control of the draft tube flow in a part-load francis turbine, J. Fluid Engineering 131 (2009) $1-13$

[5] S. Tridon, G.D. Ciocan, S. Barre, L. Thomas, 3D timeresolved PIV measurement in a francis turbine draft tube, proceedings of 24st IAHR Symp, on Hydraulic Machinery and Systems, Foz Do Iguassu, Brazil, 2008

[6] C. Fureby, G. Tabor, H.G. Weller, A.D. Gosman, A comparative study of subgrid scale models in homogeneous isotropic turbulence, Phys. Fluids 9 (1997) 233-258

[7] A. Yoshizawa, Statistical theory for compressible turbulent shear flows, with the application to subgrid modeling, Phys. Fluids 29 (1986) 2152-2164

[8] I. Orlanski, A simple boundary condition for unbounded flows, J. Comput. Physics 21 (1976) 251-269

[9] C. Duprat, G. Balarac, O. Métais, Wall boundary conditions in Large-Eddy Simulation for high Reynolds number flow with pressure gradient, proceedings of Euromech colloquium 504, Technische Universität München, Germany, 2009

[10] E. Balaras, C. Benocci, U. Piomelli, Two-layer approximate boundary conditions for large-eddy simulation, AIAA J. 34 (1996) 1111

[11] M. Manhart, N. Peller, C. Brun, Near-wall scaling for turbulent boundary layers with adverse pressure gradient, Theor. Comput. Fluid Dyn. 22 (2008) 243-260

[12] M.J. Nituch, S. Sjolander, M.R. Head, An improved version of the cebeci-smith eddy-viscosity model, Aeronautical Quarterly 29 (1978) 207-225

[13] C. Duprat, O. Métais, Large-Eddy Simulation of a high Reynolds number swirling flow in a conical diffuser, proceedings ERCOFTAC WORKSHOP Direct and Large Eddy Simulation 7, Trieste, Italy, september 8-10, 2008

[14] M.J. Landman, On the generation of helical waves in circular pipe flow, Phys. Fluids 2 (1990) 738-747 